\title{
A Generic Transformation of Advanced Materials Science and Technologies: Towards Multi-Functional and Intelligent Multi-Materials Systems
}

\author{
Tarık Baykara* \\ Department of Mechanical Engineering, , Turkey \\ *Corresponding author: Tarık Baykara, Department of Mechanical Engineering, Doğuş İstanbul, Turkey

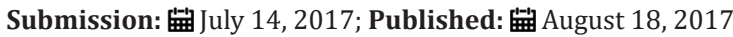

\begin{abstract}
Materials technologies are considered to be one of the major pillars and backbones of modern society with other generic fields such as energy, information-communication technologies and genetics \& biotechnologies. Starting with the $21^{\text {st }}$ Century, a new era for advanced materials has emerged and greatly influenced by the market dynamics and intense competition from new entries (e.g China and other new competitor countries). Advanced materials engineering has been evolving to become significantly more "integrated materials systems" towards more multi-functional and smart and intelligent. Working on individual and isolated material domains is no longer adequate enough to solve sophisticated engineering problems in industry. Therefore, a new challenge has been forcing advanced materials to become more functional, smart and intelligent and integrated with variety of other materials i.e., multi-materials systems along with interaction of other engineering functions, i.e., multi-functionality.
\end{abstract}

Keywords: Advanced materials; Integrated multi-materials systems; Innovation; Smart and functional materials

\section{Introduction}

For the last two decades, "advanced materials" have proved themselves as one of the generic, effective and strategically important key technologies. Lately, traditional categorization like "advanced ceramics", "advanced metals and alloys", "advanced polymers" and "advanced composites" could not explain the full distinction for their applications in a very wide range of high technologies such as nanotechnologies, sensor technologies, 3-D printing technologies and others. Therefore, additional terms and labels like "high value materials", "modern materials", "future materials" and "high performance materials" are being used to describe these materials [1]. Whatever they are called, it is strongly claimed that this group of materials is the "future of every modern society" since they are the basis of all key technologies [2]. In this regard, materials technologies are also considered to be one of the major pillars and backbones of modern society with other fields like energy, genetic \&biotechnology and information\& communication technologies. Due to such characteristics and strong impacts to other fields, advanced materials are considered to be one of the generic technologies as well. Certainlly, there is a huge diversity of advanced materials based upon their properties, structures, processes and applications in a very wide range of sectoral domains. Advanced Materials' crucial role is also emphasized as one of the key enabling technologies along with photonics, nanotechnology, biotechnology and micro- and nanoelectronics and as a cross cutting advanced manufacturing systems [3].
In its classical scheme represented within the corners of a triangle (metals, ceramics and polymers) and composites along the side lines and at the center, advanced materials and its multitechnological \& multi-sectoral characteristics have unique norms and characteristics such as $[4,5]$ :

a. Research and development (R \& D) intensive;

b. Generic structure;

c. Multi-disciplinary and multi-technological;

d. High potential of cumulative effects;

e. High cost and high risk investment requirement;

f. Accelerating market potential;

g. Comparatively long term for development projects;

h. Very intense international competition.

Starting with the $21^{\text {st }}$ Century, a new era for advanced materials has emerged and greatly influenced by the market dynamics and intense competition from new entries (e.g China and other new competitor countries). Global trends represented by the terms such as "knowledge economy", "innovation \& creativity" and "smartintelligent systems" are leading advanced materials to become a critical driver for growth and prosperity for all societies. Important characteristics of new advanced materials are particularly focused 
on their technical functions and multi-faceted characteristics such as physical, mechanical, electrical, optical, chemical and other variety of properties. For many high performance applications, such unique properties along with others (smartness, eco-friendliness, light weight, high strength and durability etc.) advanced materials lead to very high added value products essential for long term profitability and market superiority for firms operating in various sectors such as machinery, manufacturing, microelectronics, transport, automotive, chemical, energy, aeronautical and other industries [6].

Rapid advancement of technologies with new scientific results and findings has started to shift advanced materials technologies from its classical scheme towards more integrated multi-materials systems via multi-functional and multi variant characteristics $[7,8]$. As the traditional disciplinary classification and descriptions fade away, recent advances indicate more integrated multi-materials systems are now far more effective [7]. The traditional classification of materials are loosing its meaning as the evolving and competitive market structures require combined and enhanced properties of variety of materials functioning within a system's integral structure [9].

Newly formed and continously evolving norms and characteristics of advanced materials will be discussed based upon their role in newly emerging high technology applications and other challenges such as in autonomous systems. We will also adress these issues from the newly forming unique characteristics of advanced materials and some literature both on the classical and newly forming advanced materials scheme will also be outlined.

\section{A generic transformation of advanced materials technologies}

Key challenges of the $21^{\text {st }}$ Century in critical fields of energy, environment, defense \& homeland security, healthcare, transport, additive manufacturing, microelectronics, nanotechnology, space \& aviation and others are demanding high performance, enhaced properties with multi-functionality from advanced materials science and engineering. In this sense, an extensive transformation of advanced materials from traditional classification as metals, ceramics, polymers and composites should be radically changed to a newly formed, complex, integrated and dynamic system.

Such transformation of new generation advanced materials system may have the following attributes $[1,2,8,10-13]$ :

a. High-tech applications

b. High value-added products

c. Multidisciplinary (physics, chemistry, applied mathematics, biology, mechanical, electrical-electronical engineering and others)

d. Multitechnological (biosciences, micro-electronics, nanoelectronics, photonics, mechatronics, machinery and others)

e. Multisectoral (energy, transport, medical-healthcare, sports, food packaging, space $\&$ aviation, civil engineering and others)

f. Multi-functionality (mechanical, physical, chemical, electrical and others)

g. Intelligence and smartness

h. Miniaturization; smaller length scales towards nanosystems

In its historical context, materials industry was largely based on substitution of natural materials with synthetic, man-made materials during the first half of the $20^{\text {th }}$ Century (e.g., leather by polymeric materials, cotton by synthetic fibers, wood by iron-steel etc.). In the second half of the previous century, materials technologies has shifted from substitution to custom-made materials inquired by the industry (e.g. electronic ceramics for microelectronics, polycarbonate for compact discs etc.). This shift greatly exploited scientific and technical advances following the $2^{\text {nd }}$ World War and brought tremendous advantage and improvement for newly rising industries such as microelectronics, communication, machinery, transport, space \& aviation and others. During the last couple of decades, another concept has evolved based upon the needs and necessities of revolutionary market dynamics particularly in the fields of biotechnology, information technologies, nanotechnologies and cognitive systems simply stated as "bio-info-nano and cogno systems". Other than scientific and technological advances in design, processing, production and quality, governmental policies, market conditions and societal needs and requirements were also effective for shaping and leading advanced materials technologies forward to reach beyond the $21^{\text {st }}$ Century $[2,14]$.

Starting with the late 90 s and during the dawn of the new century, emerging scientific and technical advances such as high capacity computational modelling of atoms and molecules to design and tailor new and original compounds for sophisticated functions, rapid advancement of nanosciences and nanotechnologies along with the development of highly capable and effective analytical instruments for testing, analysis and characterization of microelectronics, nanostructures (e.g. ultrahigh resolution electron microscopes) lead to a new era for advanced materials technologies. Such a large shift within the materials technology is demonstrated in (Figure 1).

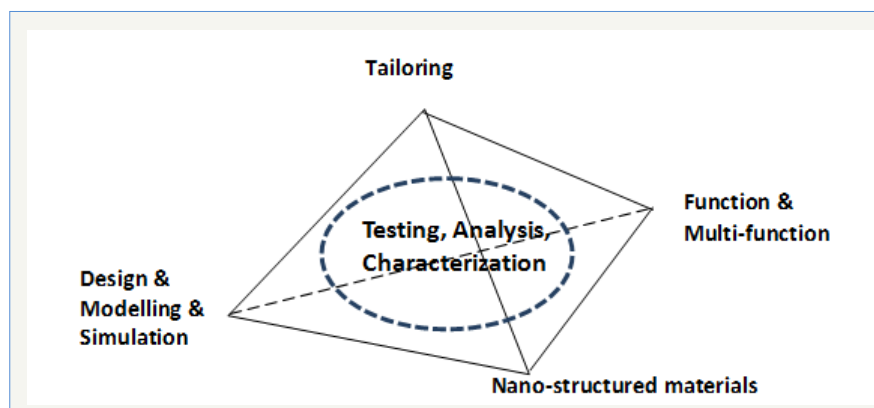

Figure 1: New relationship within the design of novel materials for $21^{\text {st }}$ century.

This brings newly formed and evolving norms and characteristics for advanced materials such as follows: 
a. Rapidly decreasing size of materials leading nanostructured materials within atomic/molecular dimensions; it should be noted that nanosciences at atomic/molecular level bring unusual novel properties and changing characteristics.

b. Based on these developments, processing, development, testing and fabrication are also getting into the nano level science and technology which bring radical changes in almost whole aspects of materials engineering.

c. Accelarating accumulation of knowledge is leading new and quantum-based data storage, communication and diffusion of new techniques and capabilities. New analytical techniques (e.g. high resolution electron microscopy techniques and other advanced imaging and manipulation techniques) and methods in testing, analysis and characterization of materials' properties cause in-depth understanding of atomic and molecular interactions leading new and novel materials synthesis.

d. Already existing characteristics of multi-disciplinarity and multi-technological features lead to new emerging fields such as bio-materials, magneto-optical materials, nano-materials, smart and intelligent materials and many others.

e. Based upon such emerging characteristics, advanced materials engineering has been evolving to become significantly more "integrated materials systems". Working on individual and isolated material components is no longer adequate enough to solve sophisticated engineering problems in industry. Therefore, a new challenge has been forcing advanced materials to become more integrated with variety of materials i.e., multimaterials systems along with interaction of other engineering functions, i.e., multi-functionality.

New era is strongly demanding complex and dynamic attributes such as innovation and creativity, rapid deployment and commercialization, extensive collaboration in R\&D which is becoming R\&D\&I (innovation), efficient processing techniques for high quality products [15]. Therefore, a new and emerging relationship within the design of advanced materials engineering is evolving and shown in (Figure 1).

\section{New characteristics of advanced materials technologies}

New advanced materials technologies have been considered as key drivers for profitability and growth in $21^{\text {st }}$ Century's fast changing environments and severe competiton with the new entrants such as China, India and other Asian and Latin American countries (Brasil, Mexico) [16,17]. Today's high risk, and uncertain market circumstances is demanding new and significantly different novel characteristics. Firstly, innovation and creativity in advanced materials technologies are becoming major cornerstones for almost any organizations. In this regard, collaborative networking in all levels of industrial operations is emerging as a new paradigm for advanced materials [18]. Other than scientific and technological advances in design, processing, production and quality, governmental policies, market conditions and societal needs and requirements are also effective for shaping and leading advanced materials technologies forward [14]. R\&D intense environment is shifting towards more on R\&I (innovation) based operations and R\&I is becoming a fundamental economical activity within organizations. It should be noted that more than half of all technical innovations in practically all technology sectors and branches of industry based upon the properties of the materials in use in varying degrees [19].

Recently accelerating additive manufacturing technologies would be a new and challenging opportunities for advanced materials innovation. A limited use of polymers and some alloys is required to be improved through tailored, multi-functional, functionally graded multi-materials, nano-structured materials with variety of properties. Revolutionary improvements are expected in this technology through enabling materials such as energy storing and conversion devices, membranes for filtration, active biomaterial implants. Development of multi-functional, functionally graded multi-materials and applying into the additive manufacturing is considered to be one of the future technologies using optimised designs to control microstructure, properties, processes and performances of novel systems [20].

As one of the key enabling technologies, advanced materials applications have been evolving into new level of understanding with the following developments in materials science and technologies (Figure 1) [3,21]:

a. Tailoring: Unique, newly created enabling materials, products.

b. Improvements in new and enabling manufacturing processes.

c. Enhancement in already existing products through newly formed properties, processes and forms

d. Design, Modelling, Simulation: Rational design strategies and their integration into structures and systems; in such a material system, designing smart structures with multifunctional concepts (e.g. self-sensing, self-healing etc.).

e. Nano-structured materials for multi-functional applications.

f. Multi-functional, multi-purpose, high performance printing materials for 3-D printing technologies [22].

Materials R\&D\&I (Research\&Development\&Innovation) is a crucial source of innovation and enhances competitiveness in many key technologies. Key enabling technologies including micro/ nanoelectronics, additive manufacturing, energy, space, robotics, biomedical and other technologies are widely dependent upon materials R\&D\&I. As pointed before, advanced materials sector is largely $R \& D$ intensive due to its nature and $R \& D$ activities in advanced materials inquire heavy investment with high cost and usually take place in universities, government laboratories and in laboratories of large firms and corporations [9]. Other than basic and applied R\&D activities in advanced materials, emerging demand for commercialization process inquires extensive R\&D\&I 
including prototype development, pilot plant applications and demonstrative process\&product developments along with variety of specific customer demands such as unique testing procedures, specialty design needs for product \& process attributes. The whole R\&D stage should be planned towards effective commercialization which are extremely expensive and needs custom-designed R\&D processes.

Such R\&D process should have the following norms and characteristics:

a. The whole research activities should be shaped upon the specific demands, inquiries and necessities of the customer. A detailed plan and unique processing including standard and non-standard testing procedures from the start to the end should be carefully designed and coordinated with customers.

b. Such customized R\&D should eliminate technological uncertainties in the planning stage and an extensive and close interaction with the customer should be coordinated effectively. Specific market norms and characteristics should be taken into account in developing products and processes with the accepted qualifications.

c. Customized R\&D activities should be conducted with multi-disciplinary expert teams including scientists, engineers, research specialists and capable technical support personel. In many advanced materials research projects, experts from the fields of chemistry, chemical engineering, physics, mechanical engineering, microelectronics, electromagnetics, software development and others are involved in various steps during the projects.

d. A team of market specialists should also be involved during the project activities for more effective commercialization along with IP specialists.

Regularly developing new technical and scientific achievements and improvements in advanced materials cover newly forming characteristics in diverse and multi-sectoral markets.

Such factors can be classified within the following basic headlines as:

i. Innovativeness \& Creativity

ii. Advanced materials as integrated multi-materials systems

iii. Collaboration \& Networking

iv. Critical performance \& Multi- and/or special-functionality

v. Challenge of commercialization

Newly evolving concept of "commercialization" inquires a wholly new understanding of mechanisms within the market, organization and processes such as non-lineer perceptions and chaotic system thinking and others. In this regard, more combinatorial (and hybrid) materials systems are modelled through sophisticated software programmes and simulation techniques leading to tailored properties upon customers' demands. The mechanism of innovation and commercialization for advanced materials have a complex and dynamic nature and couldn't be categorized in one of the following models: "market pull" or "technology push" mechanisms. New molecules designed through complex computational simulations and recent hybrid processing techniques are highly advanced and unknown for markets and customers. In this regard, a plane "market pull" mechanism is hard to be operative in many instances. On the other hand, "technology push" mechanism has difficulties in commercialization of any innovative ideas due to high risk, uncertainties and many other complexities. It may take a long delay for any new material innovation to be transferred into a viable product and full commercialization. In this mechanism, high cost and extended duration of research, development and time lag for any product to reach the market are also considered as barriers. Based upon these limitations and problems, the "TechnologyMarket Matching (coupling)" mechanism has a very high potential in this respect to find new business models and collaborative results in commercialization $[9,23]$. As indicated before, since advanced materials technologies carry challenges both in market and in technological (high risk, uncertainties, long duration of research, high cost of investment etc) innovation mechanism is categorized along the market-technology matching (coupling) model.

The majority of customers' demands and desires for materials (for both products and processes) can be listed as follows:

a. Decreasing size and weight is the predominant inquiry;

b. Almost all organizations desire to be innovation-centric and seek for creative solutions in all levels of operations.

c. Functionality within the same material's platform is a must and integrity is primarily demanded.

d. High value added products are the major targets of organizations.

e. Lowering cost and high volume production are secondary concerns.

f. Collaborative networking is extensively emerging and many firms are seeking for reliable and efficient university collaborations.

g. Major areas of interests in advanced materials: Tailored materials; Smart / Intellectual systems; Nano-structured materials (as functional coatings); Functional materials systems.

\section{Conclusion}

A new approach and understanding for advanced materials complex depicted as "integrated materials systems" may reflect the following attributes:

A. In such materials complexes, one may not consider materials as simple and plane elements and/or sub-elements of a system. The system itself is a new advanced materials platform as an integral entity developed for specific functions with multi-variant properties and characteristics. 
B. Emerging new models for advanced materials are based upon combining and putting all together the variety of subfields of materials science and many other engineering fields (chemical, mechanical, microelectronics, software etc) to solve the challenging problems with such "integrated systems materials engineering".

C. Design, Modelling, Simulation: Rational design strategies and their integration into structures and systems; in such a material system, designing smart structures with multifunctional concepts (e.g. self-sensing, self-healing etc.).

D. Revolutionary improvements are expected in 3-D printing technology through enabling enabling materials such as nano-structured materials, smart materials, multi-functional materials. Development of multi-functional, functionally graded multi-materials and applying into the additive manufacturing is considered to be one of the future technologies using optimised designs to control microstructure, properties, processes and performances of novel systems.

E. Such a complex materials system may not be developed by single and/or limited actors, an extensive collaborative networks should be developed for innovative materials system. Actors of networks should include a range of actors from varying fields and sectors.

F. University, academia involvement must be an unseperable actors for such networking for the scientific and technological framework forthe development of new advanced composites.

G. High risk and high cost of investment and maintenance for processing, testing, analysis and characterization during the R\&D and innovation stages may be solved by directing such investments to professional research and technology organizations (RTOs). Such RTOs may play a central role in variety of networks as service providers in processing, testing, analysis and characterization along with other tasks such as sub-contracting and consulting as well as collaborative partners.

\section{References}

1. Charles F, Eoin O'Sullivan (2014) A review of international public sector strategies and roadmaps: A case study in advanced materials. Entre for Science Technology and Innovation, Institute for Manufacturing, University of Cambridge, UK.

2. Dosch H, Van de Voorde MH (2001) Materials science and basic research in Europe: Conclusions and reccomendations Chapter 9, European White Book on Fundamental Research in Materials Science, Max-PlanckGesellschaft, Germany.

3. Richter H (2011) Materials for Key Enabling Technologies European Science Foundation, Materials Science and Engineering, Expert Committee (MatSEEC).

4. Baykara T, Özbek S, Ceranoğlu AN (2015) A generic transformation of advanced materials technologies: towards more integrated multi- materials systems via customized R\&D and Innovation. The Journal of High Technology Management Research 26 (1): 77-87.

5. Helena MM Lastres (1994) The Advanced Materials Revolution and Japanese System of Innovation, St. Martin's Press, London.

6. UK Technology Strategy Board: Driving Innovation Advanced Materials: Key Technology Area 2008-2011.

7. Yang P, Tarascon JM (2012) Towards systems materials engineering. Nat Mater 11: 560-563.

8. Deloitte Global Manufacturing Group (2012) Reigniting growth Advanced Materials Systems.

9. Maine E, Garnsey E (2006) Commercializing generic technology: The case of advanced materials ventures, Research Policy 35(6): 375-393.

10. Federal Ministry of Education and Research (BMBF) (2010) Ideas, Innovation, Prosperity: High Tech Strategy 2020 for Germany BMBF Innovation Policy Framework Division, Bonn, Berlin.

11. Rooney M, Robert JC, Murray GM, Romenesko BM (2000) Advanced Materials Challenges and Opportunities. Johns Hopkins APL Technical Digest 21.

12. Leszek A Dobrzański (2006) Significance of materials science for the future development of societies. Journal of Materials Processing Technology 175(1-3): 133-148.

13. Ann Crabbéa, Ria Jacobs, VeroniqueVan Hoof, Anne Bergmansa, Karel Van Acker (2012) Transition towards sustainable material innovation: evidence and evaluation of the Flemish case. Journal of Cleaner Production 56: 63-72.

14. Bauer D, Summary Briefing (2011) US Department of Energy, Critical Materials Strategy. Workshop on Mineral Raw Material Flows and Data.

15. von der Gracht HA, Stillings C (2013) An innovation-focused scenario process-A case from the materials producing industry. Technological Forecasting and Social Change 80(4): 599-610.

16. An-ChinCheng (2012) Exploring the relationship between technology diffusion and new material diffusion: the example of advanced ceramic powders. Technovation 32(3-4): 163-167.

17. Nakagawa M, Watanabe C, Griffy Brown C (2009) Changes in the technology spillover structure due to economic paradigm shifts: A driver of the economic revival in Japan's material industry beyond the year 2000. Technovation 29(1): 5-22.

18.Valk, Vander TC, MMH Gijsbers, GW (2011) Evaluating innovation networks in emerging Technologies. Technological Forecasting and Social Change 78(1): 25-39.

19. Ehrenfried Z (2012) Advanced Materials, R\&D Cooperation for Innovation Products, Aarhus, Denmark.

20. Grant P (2013) New and advanced materials, UK Government's foresight future of manufacturing project.

21. Faulkner A, Berenshteyn Y (2013) Advanced materials: Creating chemistry between innovators and investors. Cleantech Group.

22. Lewis JA, Scott CS (2012) Printing Functional Materials. Harvard School of Engineering and Applied Sciences Wyss Institute for Biologically Inspired Engineering.

23. Maine E, Probert D, Ashby M (2005) Investing in new materials: A tool for technology managers. Technovation 25(1): 15-23. 